\title{
Advances in Processing, Manufacturing, and Applications of Magnetic Materials
}

\author{
S.K. MCCALL (10) ${ }^{1,3,4}$ and I.C. NLEBEDIM $\mathbb{1}^{2,3,5}$ \\ 1.-Lawrence Livermore National Laboratory, Livermore, CA 94551, USA. 2.-Ames \\ Laboratory, Ames, IA 50011, USA. 3.-Critical Materials Institute, Ames, USA. \\ 4.—e-mail: mccall10@llnl.gov. 5.—e-mail: nlebedim@ameslab.gov
}

Magnetic materials are increasingly important for many green energy technologies. Probably, the best known of these are permanent magnets. They are used to supply a magnetic field and are widely used in actuators, motors, generators, data storage, and sensors. These are "hard magnets," meaning that they retain a large permanent magnetization (difficult to be demagnetized), which is what makes them so useful in motors where an opposing magnetic field can be used to push against them. A coercivity (resistance to demagnetization) of $\sim 400$ Oe $(32,000 \mathrm{~A} / \mathrm{m})$ or more is typically the threshold value for a hard magnet and is typical of isotropic sintered ferrite $\left(\mathrm{SrFe}_{12} \mathrm{O}_{19}\right)$ such as found in many "refrigerator magnets." Comparably important soft magnets are useful for directing magnetic fields and can be very easily (de)magnetized. As such, they typically have a coercivity $<12.5 \mathrm{Oe}$ $(1000 \mathrm{~A} / \mathrm{m})$, and specialized very soft magnets can have coercivities $<0.00125 \mathrm{Oe}(1 \mathrm{~A} / \mathrm{m})$. Common applications include coil cores, transformer cores, and magnetic shielding. Additionally, they are important for many AC electrical applications such as inductors, filters, and resonators, particularly at high frequencies up to and including microwaves.

In addition to the composition of these magnetic materials, controlling the microstructure is essential to producing very hard and very soft magnets. The details of the magnetic properties are highly sensitive to crystallographic orientation, grain size, and defects, thus processing can be as important as the material chemistry. Advances in manufacturing

S.K. McCall and I.C. Nlebedim are Guest Editors for the Nuclear Materials Committee of TMS and coordinated the topic Advances in Processing, Manufacturing, and Applications of Magnetic Materials in this issue.

(Received October 5, 2021; accepted October 5, 2021;

published online October 21, 2021) and new approaches to processing offer opportunities to improve performance of magnetic materials. This issue of JOM contains a set of papers exploring some new approaches to manufacturing of magnetic materials, computational insights, and the challenges in producing these materials with the desired properties.

Donghui Liu et al., in "Preparation and Magnetic Properties of 2:17-type SmCo Alloy by Transition Metal-induced Calciothermic Reduction," describe a single-step method for producing an alloy based on the $\mathrm{Sm}_{2} \mathrm{Co}_{17}$ structure produced through a singlestep calciothermic reaction where the resulting alloy is an admixture of the $\mathrm{SmCo}_{7}$ and $\mathrm{Sm}_{2} \mathrm{Co}_{17}$ phases. A key advance is the use of $\mathrm{SmF}_{3}$ in lieu of $\mathrm{Sm}$ metal in the synthesis process, which avoids the need to separately reduce $\mathrm{Sm}$ to metal and the associated challenges of protecting and transporting a highly reactive metal from the metal producer to the magnet maker. The post-processing steps result in a very fine microstructure and quite good magnetic properties, with an intrinsic coercivity of $\sim 27 \mathrm{kOe}(2100 \mathrm{kA} / \mathrm{m})$ and an energy product above 25 MGOe $\left(200 \mathrm{~kJ} / \mathrm{m}^{3}\right)$, comparable with many commercial Sm-Co based magnets.

Permanent magnets based on $\mathrm{SmCo}_{5}$ with high coercivity have found significant application especially where resistance to demagnetization outweighs the amount of magnetic flux needed. Driven by the need to develop substitutes for $\mathrm{SmCo}_{5}$ magnets, Huseyin Ucar and Durga Paudyal presented a computational study proposing the replacement of Sm with Ce and $\mathrm{La}$ and some of the Co with Fe. In "Unraveling Site Selective Magnetic Properties of Cobalt Sites in Critical Elements Lean RE(TM)5 Magnet Materials," the authors report on two compounds, $\mathrm{CeCo}_{2} \mathrm{Fe}_{3} \mathrm{~N}_{2}$ and $\mathrm{LaCo}_{2} \mathrm{Fe}_{3} \mathrm{~N}_{2}$, that were proposed to have sufficient magnetic hardness for permanent magnet development. The key approach is to place Fe (an abundant and inexpensive element) in positions where the 
contribution of Co (a more expensive element) to magnetic hardness is minimal, while using nitrogen to stabilize the compounds.

Considering soft magnets, Bowen Dong et al. describe an inexpensive sol-gel synthesis route for core-shell ferrite powders where a metallic FeCo core is coated by an insulating $(\mathrm{Mn}, \mathrm{Zn})$ ferrite shell in their paper "Field Assisted Sintering of $\mathrm{FeCo} /$ MnZn Ferrite Core-Shell Structured Particles." The resulting composite powders were consolidated using a field-assisted sintering technique. The resulting materials have saturations comparable to silicon steels with the potential to have significantly lower eddy current losses at high frequencies due to the high resistivity of the ferrite shells. The ability to operate at higher frequencies enables higher speed motors (30-1000 kRPM), which leads to increased power densities and miniaturization providing opportunities for reduced material intensity as well as improved energy efficiency.
This work was supported by the Critical Materials Institute, an Energy Innovation Hub funded by the US Department of Energy, Office of Energy Efficiency and Renewable Energy, Advanced Manufacturing Office. Work prepared by LLNL under Contract DE-AC52-07NA27344 and was partially supported by the LLNL-LDRD Program under Project No. 20-ERD-059 and by Ames under contract DE-AC02-07CH11358.

All titles and authors of the articles published under the topic "Advances in Processing, Manufacturing, and Applications of Magnetic Materials" in the December 2021 issue (vol. 73, no. 12) of JOM can be accessed via the journal's page at: http://link.sp ringer.com/journal/11837/73/12/page/1.

Publisher's Note Springer Nature remains neutral with regard to jurisdictional claims in published maps and institutional affiliations. 